\title{
Distal Pectoralis Major Tears
}

\section{Sonographic Characterization and Potential Diagnostic Pitfalls}

\author{
Sun Joo Lee, MD, Jon A. Jacobson, MD, Sung-Moon Kim, MD, David Fessell, MD, Yebin Jiang, MD, PhD, \\ Gandikota Girish, MD, Olaf Magerkurth, MD
}

Received April 24, 2013, from the Department of Radiology, University of Michigan Medical Center, Ann Arbor, Michigan USA (S.J.L., J.A.J., S.-M.K., D.F., Y.J., G.G., O.M.); Department of Radiology, Inje University College of Medicine, Busan Paik Hospital, Busan, Korea (S.J.L.); and Department of Radiology, University Children's Hospital, Basel, Switzerland (O.M.). Manuscript accepted for publication May 17, 2013.

This work was supported by the 2012 Inje University research grant.

Address correspondence to Jon A. Jacobson, $M D$, Department of Radiology, University of Michigan Medical Center, 1500 East Medical Center Dr, TC2910L, SPC 5326, Ann Arbor, MI 48109 USA.

E-mail:jjacobsn@umich.edu

Abbreviations

MRI, magnetic resonance imaging
Objectives-Distinction between musculotendinous tears of the pectoralis major and distal tendon avulsions is important, as the latter typically requires surgical repair. The objective of this study was to characterize the sonographic appearances of surgically proven distal tendon avulsion tears of the pectoralis major.

Methods-A retrospective search of the radiology database (2001-2011) revealed 22 cases of pectoralis major tears on sonography, of which 9 had surgical correlation. Sonograms were retrospectively characterized by 3 fellowship-trained musculoskeletal radiologists in consensus with respect to the location and size of the fluid collection and the presence of tendon or tendonlike tissue superficial to the biceps brachii tendon.

Results - At surgery, complete distal tendon avulsions or tears of the sternal head of the pectoralis were present in all 9 cases: isolated in 6 and combined with clavicular head tears in 3 . The location of the fluid collection was at the musculotendinous junction in $89 \%$ ( 8 of 9 ) and medial to the biceps brachii tendon in $11 \%$ ( 1 of 9 ), with a mean largest dimension of $3.8 \mathrm{~cm}$ (range, $0.7-6.2 \mathrm{~cm}$ ). In no case was fluid seen at the humeral attachment of the pectoralis. In 67\% (6 of 9), linear thickened hypoechoic tissue was seen superficial to the biceps brachii tendon, which simulated an abnormal but intact tendon, whereas in 33\% (3 of 9), a normal distal pectoralis tendon was seen.

Conclusions - Surgically proven distal pectoralis major tendon avulsions most commonly showed fluid collections at the musculotendinous junction and not at the humeral shaft, with either a normal tendon or hypoechoic tendonlike tissue over the biceps brachii long-head tendon. These findings may potentially cause misdiagnosis of distal tendon avulsions or tears as musculotendinous injuries.

Key Words - injury; magnetic resonance imaging; pectoralis; sonography

I njuries of the pectoralis major muscle are important, as they can result in functional and cosmetic deficiency of the upper extremity. The most common mechanism of injury is indirect, resulting from sudden forceful overload applied to a maximally contracting muscle, ${ }^{1}$ as seen with activities such as weight lifting, wrestling, football, and water skiing. ${ }^{2}$

The management of pectoralis major muscle injuries is based on an accurate assessment of the extent and site of injury. The extent of injury is classified as muscle contusion and strain, a partial tear, and a complete tear. Partial injuries are almost uniformly treated with conservative management. Injuries are further subdivided according to location: muscle, myotendinous junction, and tendon. 
The location of the tear is important because tendon avulsion from the humerus is treated with rapid surgical repair, whereas myotendinous injuries are usually treated in a conservative manner. ${ }^{2,3}$ Because an accurate clinical assessment of pectoralis major muscle injuries may be difficult, imaging is commonly needed.

Magnetic resonance imaging (MRI) has been used with success to characterize pectoralis injuries. ${ }^{4,5}$ Similarly, sonography has also been used to diagnose and characterize the extent and site of a pectoralis injury. ${ }^{6}$ However, accurate imaging diagnosis may still be problematic given the complex anatomy of the distal pectoralis tendon, which involves a $180^{\circ}$ twisting of the distal tendon, composed of clavicular and sternal heads.

In our clinical practice, we have noted difficulty in the sonographic diagnosis of distal pectoralis tendon tears, especially with regard to complete distal tendon avulsions, in which we have found variability in hematoma location and distal tendon visualization. The purpose of this study was to characterize the sonographic appearances of surgically proven distal tendon avulsion tears of the pectoralis major.

\section{Materials and Methods}

Institutional Review Board approval was obtained, with informed consent waived. A retrospective search of the radiology database (2001-2011) revealed 22 cases of pectoralis major tears on sonography, of which 9 had surgical correlation. Sonographic examinations of the pectoralis major muscle were performed by 1 of 6 musculoskeletal radiologists trained and experienced in musculoskeletal sonography (range of experience, $5-15$ years). Sonograms were obtained with various ultrasound machines (iU22 and HDI 5000; Philips Healthcare, Bothell, WA; and LOGIQ 9; GE Healthcare, Milwaukee, WI) using 7-15$\mathrm{MHz}$ linear transducers.

Sonographic evaluation of the pectoralis muscle was performed longitudinal and transverse to the muscle and tendon fibers from the origin to insertion. The distal pectoralis tendon was identified and evaluated in the transverse plane at the level of the bicipital groove of the humerus, where the pectoralis tendon courses over the long head of the biceps brachii tendon. Flow was assessed on color or power Doppler imaging. Comparison to the contralateral nonaffected side and imaging with the shoulder in abduction and external rotation were at the discretion of the individual performing the sonographic examination. A normal tendon was characterized as hyperechoic and fibrillar on sonography. The normal echo pattern of the muscle in the longitudinal section consisted of fan-shaped striated echogenic lines representing septa of fibro-adipose tissue (the perimysium) against a hypoechoic background representing the muscle bundles. The thick echogenic anterior border of the muscle represents the dense connective tissue surrounding the muscle (the epimysium). Muscle fibers can be followed from the point of origin down to the musculotendinous junction, where they twist to form a fibrillar tendon that inserts onto the humerus.

Sonograms (static and cine clips when available) were retrospectively evaluated by 3 fellowship-trained musculoskeletal radiologists in consensus $(15,15$, and 6 years of experience), 2 of whom performed several of the sonographic examinations. The images were evaluated for the following: fluid collection (hypoechoic or anechoic) location (intramuscular, musculotendinous [dorsal to the deltopectoral groove ${ }^{5}$, medial to the biceps brachii tendon, or adjacent to humeral cortex), fluid collection size (largest dimension), presence of linear tendon or tendonlike tissue over the biceps brachii tendon (also characterized as hyperechoic or hypoechoic), and abnormal hypoechogenicity surrounding the biceps brachii tendon.

Patient records were also reviewed, including surgical reports. Operations were performed by 1 of 5 orthopedic surgeons with sports medicine experience. The decision to perform surgery varied and was based on clinical and imaging results, as imaging was performed as part of routine patient care.

\section{Results}

The study group consisted of 9 patients, of whom $67 \%$ (6 of 9) had full-thickness tears isolated to the sternal head at the humerus attachment, and 33\% (3 of 9) had full-thickness tears of both clavicular and sternal heads at the humerus attachment proven at surgery (Table 1). All patients were male with a mean age of 39 years (range, $21-45$ years) with $67 \%$ of the injuries (6 of 9) on the right side. With regard to the mechanism of injury, 56\% (5 of 9) were injured during a bench press while weight lifting. The other patients had an injury sustained during sports activities such as basketball, football, wrestling, and skiing. Sonograms were obtained 3 to 179 days (mean, 37 days) after injury. The surgical procedures were performed 2 to 145 days (mean, 31 days) after the sonographic examinations.

At retrospective evaluation, the location of the fluid collection was at the musculotendinous junction in $89 \%$ (8 of 9) (Figure 1) and medial to the biceps brachii tendon in $11 \%$ ( 1 of 9 ) (Figure 2). In no case was fluid seen intramuscularly or at the humeral attachment of the pec- 
toralis. The mean largest dimension of the fluid collection was $3.8 \mathrm{~cm}$ (range, $0.7-6.2 \mathrm{~cm}$ ). In 33\% (3 of 9), a normal distal pectoralis tendon (linear and hyperechoic) was seen attaching to the humerus (Figure 3). In the remaining 67\% (6 of 9), linear thickened hypoechoic tissue was seen over the biceps brachii tendon (Figures 1B, 2B, and 4). In no case was there complete absence of tendon or tendonlike linear tissue superficial to the biceps brachii tendon. In $22 \%$ ( 2 of 9), there was abnormal hypoechogenicity surrounding the biceps brachii tendon (Figure 1A). Four cases underwent color Doppler imaging, which showed positive flow in 3 (Figure 1D) and no flow in 1.

\section{Discussion}

In our results, sonographic findings of distal pectoralis major tendon avulsions and tears showed a fluid collection not at the humeral attachment of the pectoralis but at the musculotendinous junction and either a normal tendon or linear thickened hypoechoic tissue over the biceps brachii tendon. The amount of the fluid collection was variable (range, $0.7-6.2 \mathrm{~cm}$ ). These findings may potentially cause misdiagnosis of distal tendon avulsions and tears as musculotendinous injuries.

The pectoralis major muscle is a powerful internal rotator, flexor, and adductor of the arm and has a broad origin from the clavicle, sternum, and cartilages of the first 6 ribs. The muscle fibers of the pectoralis major converge into 3 laminae (clavicular, manubrial, and abdominal) that rotate $180^{\circ}$ to coalesce into a tendon and are inseparably blended just proximal to their insertion onto the lateral aspect of the bicipital groove of the humerus. ${ }^{5}$ The distal tendon of the pectoralis major muscle is composed of the clavicular and sternal heads. The clavicular head consists of fibers from the clavicular lamina, which originate from the medial two-thirds of the clavicle; these fibers insert distally and anteriorly. The sternal head consists of fibers from the abdominal and manubrial laminae, which arise from the anterior surface of the manubrium, sternal body, and cartilage of the first 6 ribs; these fibers combine and course deep to the clavicular head to insert on the humerus superior to the clavicular head. ${ }^{4}$ The distal tendon of insertion on the humerus measures approximately 4 to $6 \mathrm{~cm}$ in the cephalocaudal dimension and $5 \mathrm{~mm}$ in the transverse dimension. ${ }^{5}$ As a result of the $180^{\circ}$ twist, the clavicular and upper sternal fibers insert most distally, whereas the lower sternal fibers insert most superiorly. ${ }^{5}$ The complex anatomy of the pectoralis major muscle, particularly at its insertion onto the humerus, makes imaging and diagnosis of injuries to the tendon difficult.

Patients with injuries of the pectoralis major muscle often will have pain, ecchymosis, edema, and a decreased range of motion. It is difficult to evaluate the extent and location of these injuries clinically. The incidence of traumatic injuries of the pectoralis major muscle is increasing in healthy individuals due to increasing participation in high-performance activities. Pectoralis major tendon rupture usually occurs during powerful eccentric contraction, at which time the muscle is subject to concomitant forceful stretching. ${ }^{1}$ Although injury may occur in the muscle belly due to a direct impact, ${ }^{7}$ rupture generally occurs at the myotendinous junction or the insertion onto the humerus. ${ }^{8}$ Partial tears are more common than complete tears. ${ }^{9}$ Partial tears usually occur at the myotendinous junction, whereas complete tears usually involve the distal tendon or the tendon-bone interface. ${ }^{2,4}$ Rupture of the sternal head occurs more commonly than rupture of the clavicular head, ${ }^{3}$ although in older individuals, the frequency of avulsion of the two major heads is probably the same. ${ }^{1}$ Our results are in concordance with those reported in the literature. In our study, there were isolated complete distal tendon avulsions or tears of the sternal head in 6 patients $(67 \%)$ and combined complete injury of the sternal and clavicular head in $3(33 \%)$.

Table 1. Summary of Results

\begin{tabular}{|c|c|c|c|c|c|}
\hline $\begin{array}{l}\text { Patient } \\
\text { Age, y }\end{array}$ & $\begin{array}{c}\text { Injury } \\
\text { Mechanism }\end{array}$ & Fluid Size, cm & Fluid Location & $\begin{array}{c}\text { Tissue Over Biceps } \\
\text { Brachii Tendon }\end{array}$ & $\begin{array}{l}\text { Torn Head } \\
\text { at Surgery }\end{array}$ \\
\hline 39 & Basketball injury & 1.5 & Myotendinous & Normal tendon & Sternal \\
\hline 42 & Football injury & 0.7 & Myotendinous & Thickened hypoechoic & Sternal, clavicular \\
\hline 45 & Wrestling injury & 2.2 & Myotendinous & Normal tendon & Sternal \\
\hline 21 & Bench press injury & 4.6 & Myotendinous & Thickened hypoechoic & Sternal \\
\hline 39 & Bench press injury & 5.5 & Myotendinous & Thickened hypoechoic & Sternal \\
\hline 44 & Skiing injury & 4.5 & Myotendinous & Thickened hypoechoic & Sternal, clavicular \\
\hline 38 & Bench press injury & 6.2 & Medial to biceps & Thickened hypoechoic & Sternal, clavicular \\
\hline 40 & Bench press injury & 4.4 & Myotendinous & Thickened hypoechoic & Sternal \\
\hline 42 & Bench press injury & 4.5 & Myotendinous & Normal tendon & Sternal \\
\hline
\end{tabular}


Treatment options for pectoralis major muscle injuries are based on an accurate assessment of the extent and site of injury. Treatment is almost uniformly nonsurgical for partial tears and occasionally for complete tears in nonathletes. ${ }^{10,11}$ Surgical repair is used for complete tears, tears involving the distal tendon, and injuries in athletes. As the sternal head of the muscle plays an important role in the function and shape of the thoracic cage, reconstruction of the torn tendon is usually performed. ${ }^{12}$ Several jeopardies of delayed diagnosis exist, including adhesions, mus- cle retraction, muscular scarring and fibrosis, atrophy, and an overall poor surgical outcome. Early surgical repair produces the best cosmetic results and functional return of full strength in young athletic individuals. ${ }^{1,3}$

Although MRI has been used to evaluate injuries of the pectoralis major muscle, ${ }^{4,5}$ sonography can also be used to characterize these injuries. ${ }^{6}$ One prior report described hypoechoic hematoma in the setting of pectoralis major tears in the elderly. ${ }^{13}$ Another report described the sonographic findings of injuries to the pectoralis major muscle,

Figure 1. Images from a 44-year-old man with complete distal avulsion tears of both pectoralis muscle heads who was injured while skiing 47 days earlier. Sonography longitudinal to the pectoralis major (A) shows fiber disruption and a fluid collection at the musculotendinous junction (arrows) with a largest dimension of $4.5 \mathrm{~cm}$. Note the hypoechogenicity surrounding the long head of the biceps brachii tendon (arrowhead). Color Doppler imaging (B) shows positive flow. Sonography transverse to the humerus (C) shows thickened hypoechoic tissue (arrows) over the long head of the biceps brachii tendon (arrowhead). Evaluation of the asymptomatic contralateral side transverse to the humerus (D) shows an intact distal pectoralis major tendon (arrows). Arrowhead indicates the long head of the biceps brachii tendon. CB indicates coracobrachialis muscle; and H, humerus.

A

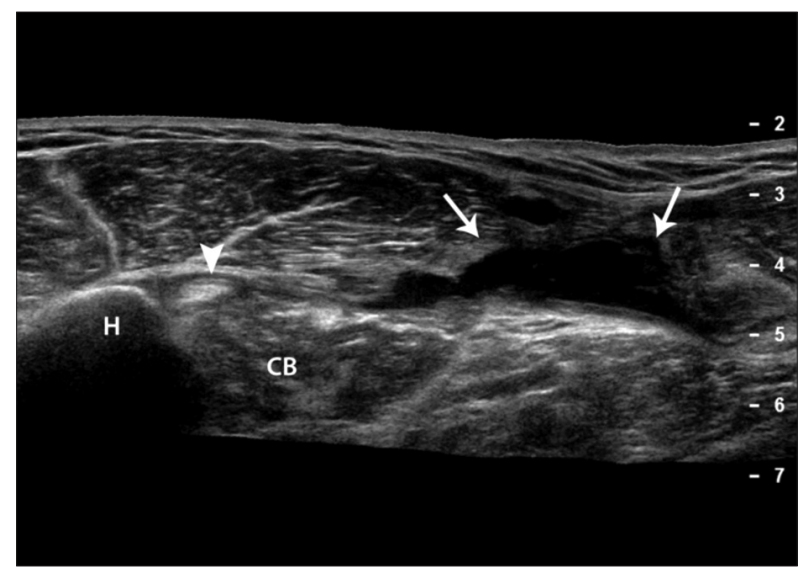

C

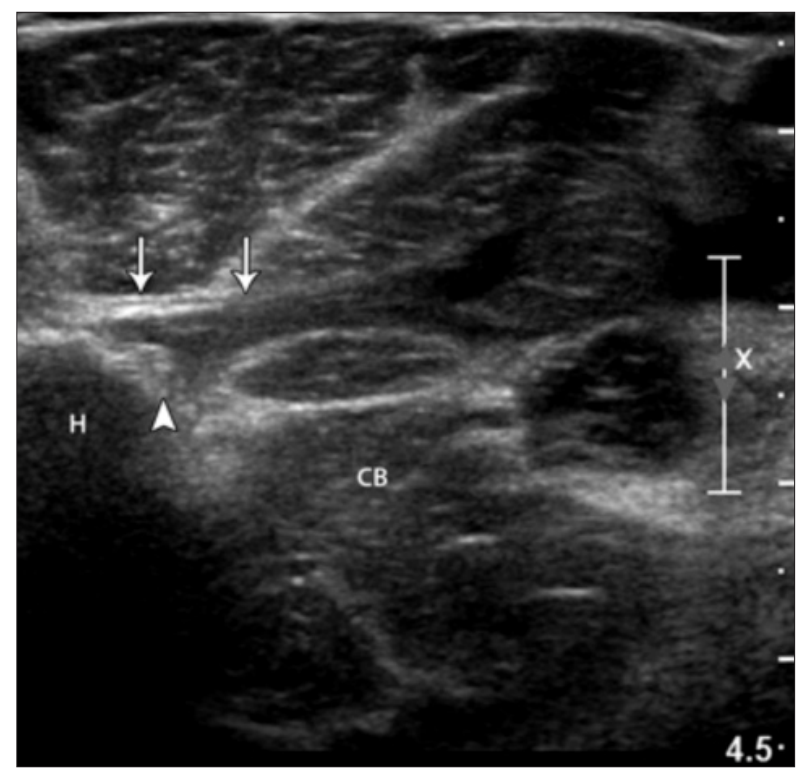

B

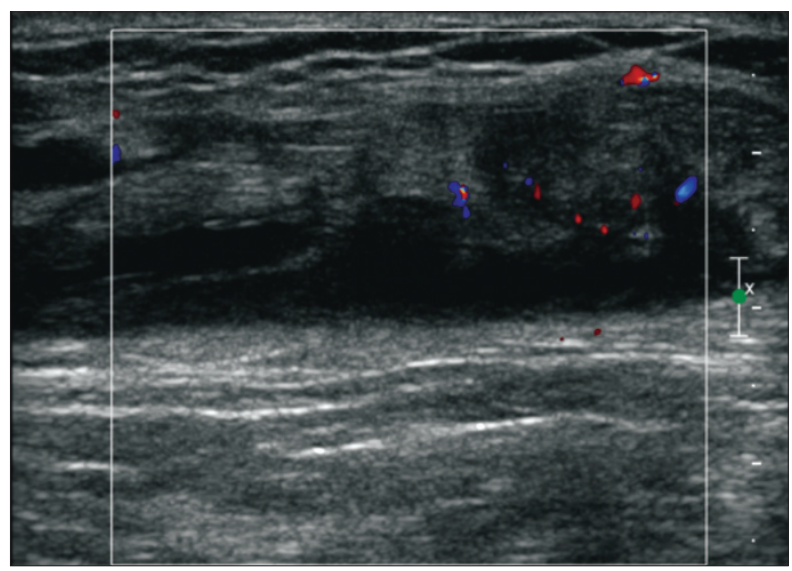

D

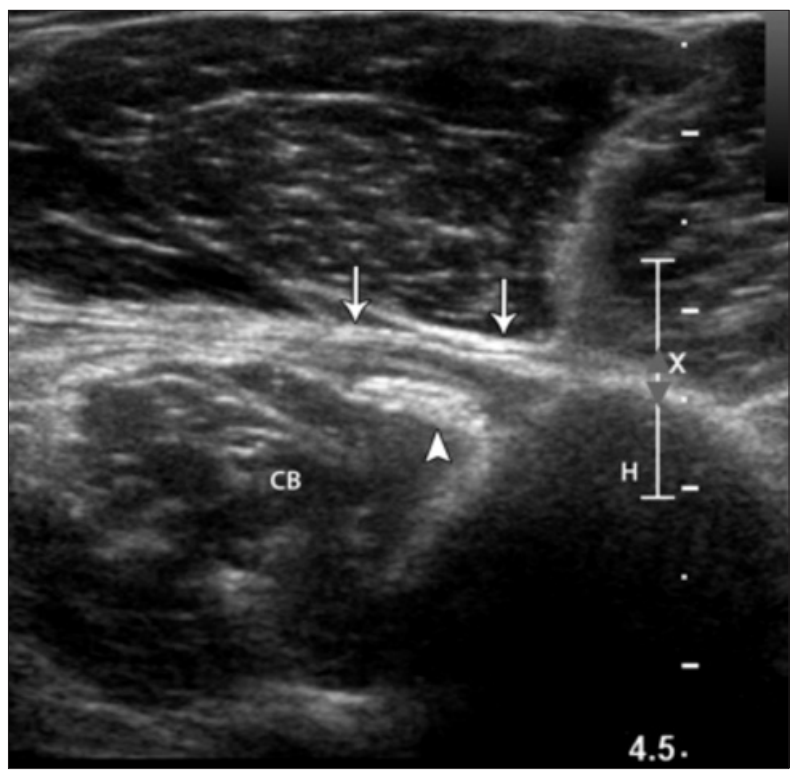


including disruption of the musculotendinous junction, muscle fiber retraction, and possible surrounding anechoic or hypoechoic hemorrhage. ${ }^{6}$ Although this latter study described the importance of distal pectoralis major tendon visualization superficial to the biceps brachii long-head tendon in the differentiation between distal and musculotendinous injury, we found such differentiation difficult in our clinical practice, which was the premise for our study. ${ }^{6}$

In our results, most cases of distal pectoralis major tendon avulsions or tears were associated with a fluid collection not at the humeral attachment of the pectoralis but at the musculotendinous junction, which could potentially cause misdiagnosis of distal tendon

A

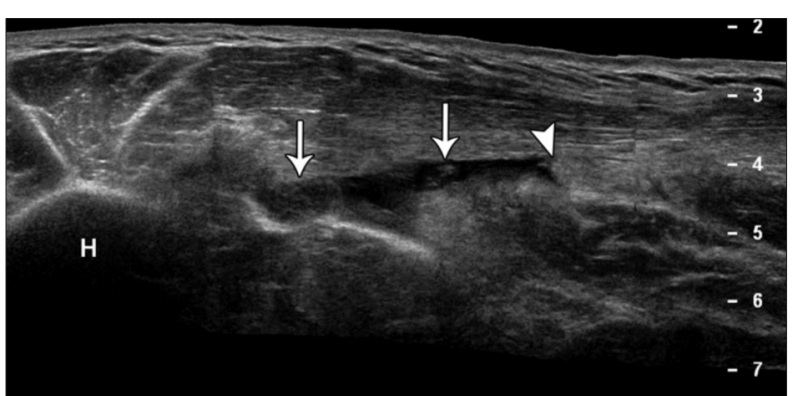

B

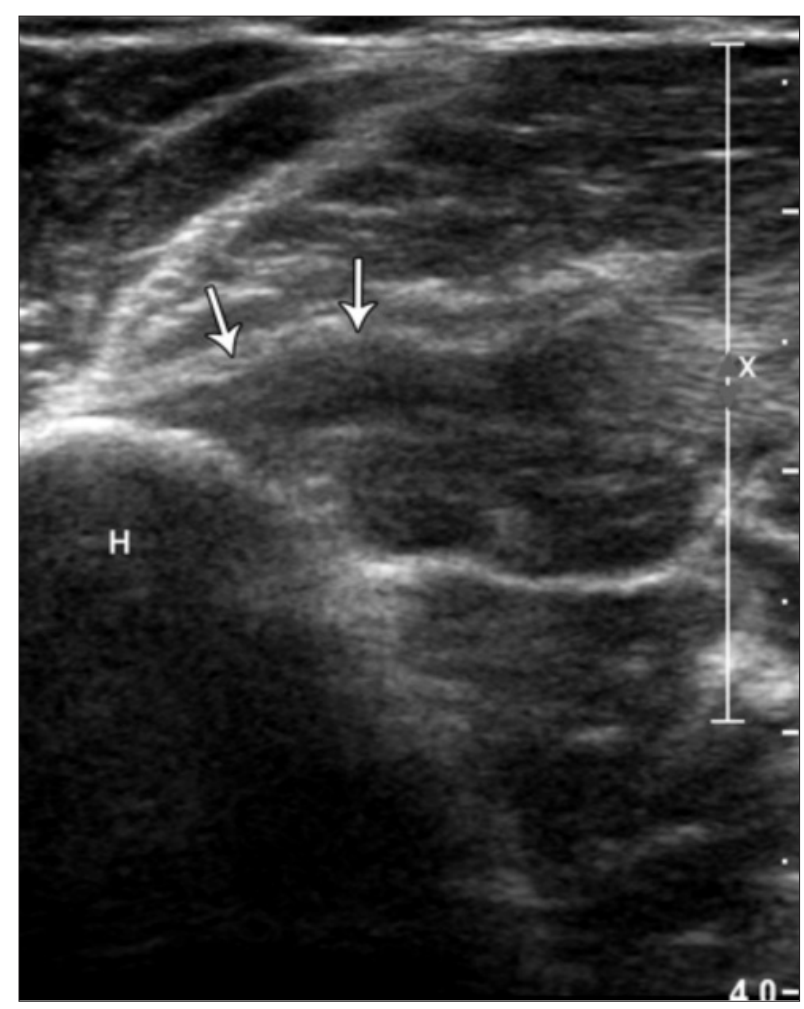

avulsions and tears as musculotendinous injuries. In only 1 case was a fluid collection seen medial to the biceps brachii tendon. The amount of the fluid collections was variable; the range in size was most likely related to the interval between injury and sonography and the severity or pattern of injury. The location of the fluid collection or hematoma in each case may be explained by the considerable retraction associated with full-thickness pectoralis tendon tears.

In $67 \%$ of our patients ( 6 of 9 ) with surgically proven distal pectoralis tendon tears or avulsions, linear thickened hypoechoic tissue was seen over the biceps brachii tendon, which simulated an abnormal but intact tendon.

Figure 2. Images from a 38-year-old man with complete distal avulsion tears of both pectoralis muscle heads due to bench-pressing. Sonography longitudinal to the pectoralis major (A) shows fiber disruption and retraction (arrowhead) and a fluid collection (arrows) medial to the biceps brachii tendon with a largest dimension of $6.2 \mathrm{~cm}$. Sonography transverse to the humerus (B) shows thickened hypoechoic linear tissue (arrows). Evaluation of the asymptomatic contralateral side $(\mathbf{C})$ shows a normal distal pectoralis major tendon (arrows). H indicates humerus.

C

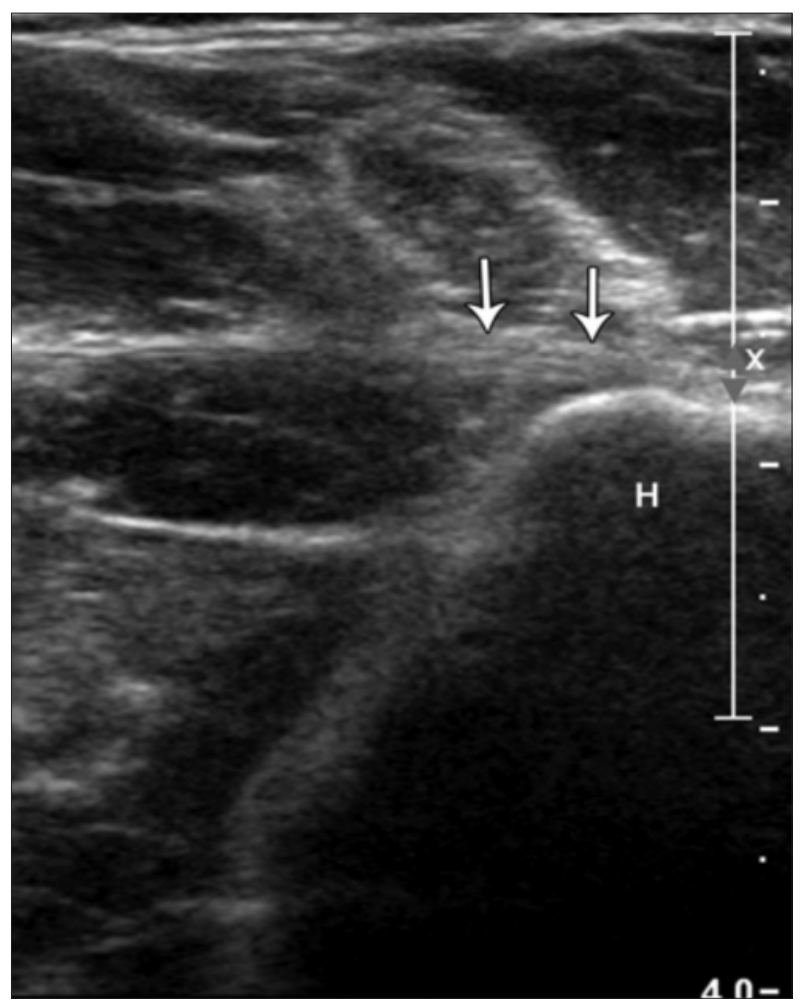


This finding in the setting of a distal and retracted tear may be from collapsed tissue planes, fibrosis, scarring, and possible periosteal stripping. According to the previous literature, periosteal stripping was considered present when intermediate to increased signal intensity on MRI was seen superficial to the humeral cortex at the insertion site in the presence of tendon avulsion. ${ }^{4}$ In addition, chronic injuries manifested as low signal intensity on MRI, findings indicative of scarring and fibrosis, which may have contributed to the sonographic findings. ${ }^{4}$ In $33 \%$ (3 of 9), a normal distal pectoralis tendon was seen attaching to the humerus. This feature was only shown in isolated sternal head injury, which may be due to the complex anatomy of the distal pectoralis tendon, where the normal fibrillar echo texture of the clavicular head could be misinterpreted as intact distal tendon fibers of the sternal head. This pitfall likely exists if the hematoma from an isolated sternal head

\section{A}

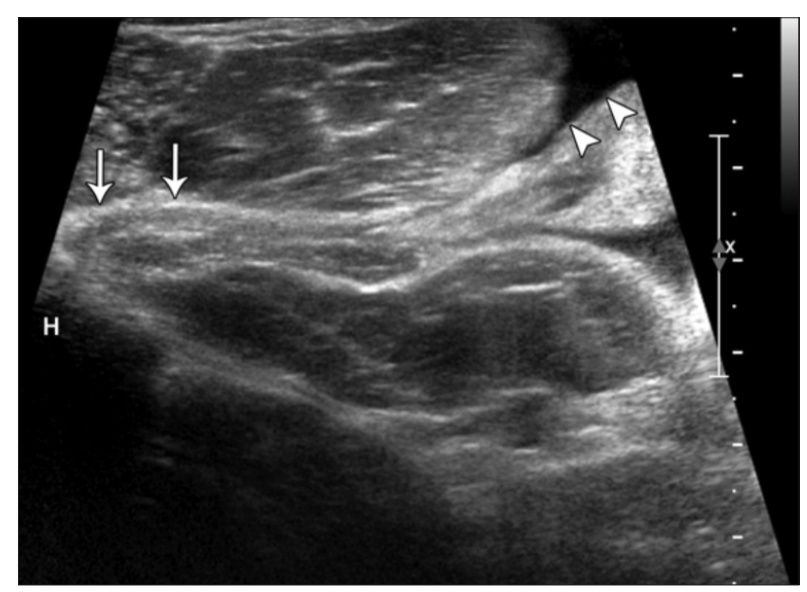

B

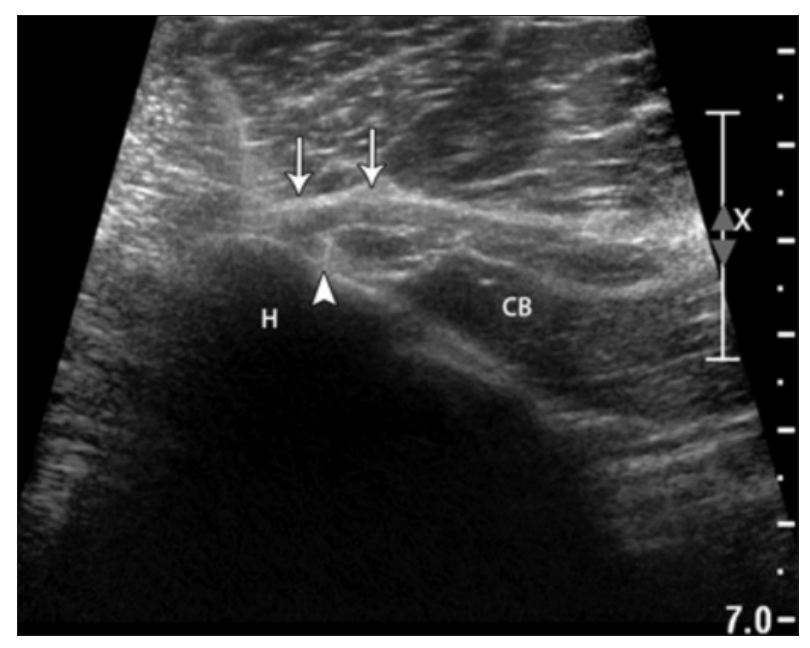

tear is in the same axial plane as the intact clavicular head tendon, which could potentially be avoided by ensuring that the most superior aspect of the pectoralis tendon, the sternal head, is selectively evaluated.

We acknowledge several limitations to this study. First, we investigated a relatively small number of 9 patients; however, the number of study participants was limited by the fact that pectoralis major muscle injuries are uncommon and the use of surgery as the standard of reference. A second limitation was the lack of comparison with surgically proven cases of injuries at the myotendinous junction or partial tears; surgical repair was used for complete tears involving the distal tendon in our institute. A selection bias was also introduced, as imaging was used in making the decisions for treatment, and the more severe injuries likely went on to surgical repair. Last, interobserver and intraobserver variability were not assessed.

Figure 3. Images from a 42-year-old man with an isolated distal tear of the sternal head of the pectoralis major due to bench-pressing. Sonography longitudinal to the distal pectoralis major (A) shows fiber disruption and a fluid collection (arrowheads) at the musculotendinous junction with a largest dimension of $4.5 \mathrm{~cm}$. Note the distal pectoralis tendon (arrows). Sonography transverse to the humerus (B) shows a fibrillar echo texture of the pectoralis major tendon (arrows). Evaluation of the asymptomatic contralateral side (C) shows a normal distal pectoralis major tendon (arrows). Arrowheads in $\mathbf{B}$ and $\mathbf{C}$ indicate the long head of the biceps brachii tendon. CB indicates coracobrachialis muscle; and $\mathrm{H}$, humerus.

\section{C}

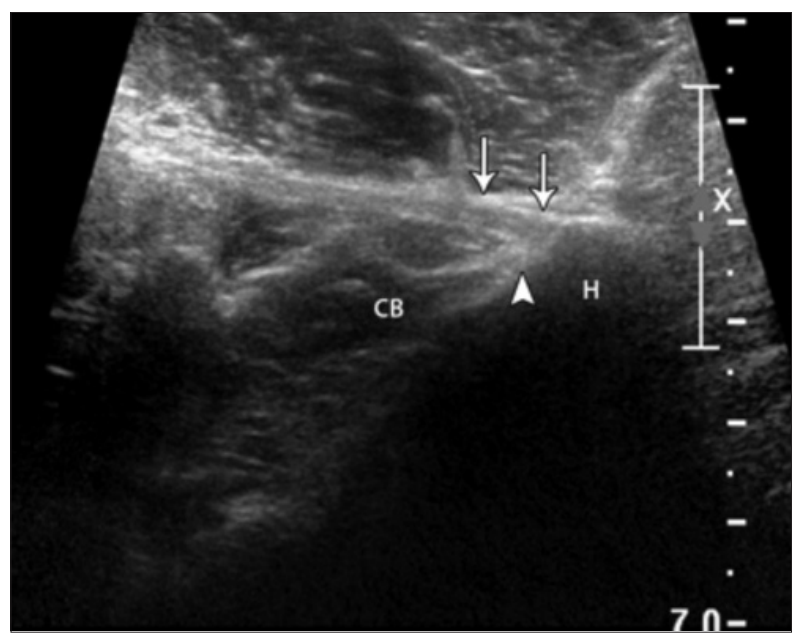




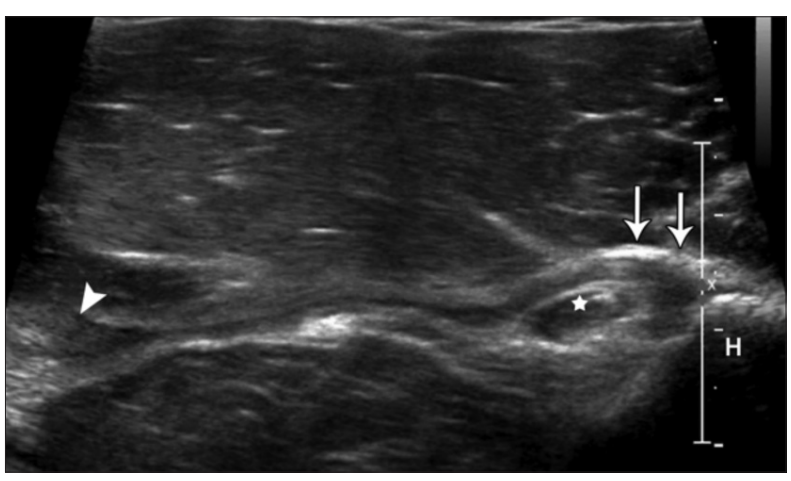

Figure 4. Image from a 40-year-old man with an isolated distal tear of the sternal head of the pectoralis major due to bench-pressing. Sonography longitudinal to the distal pectoralis major shows fiber disruption and retraction (arrowhead) and a fluid collection at the musculotendinous junction with a largest dimension of $4.4 \mathrm{~cm}$. Note the thickened hypoechoic tissue (arrows) over the biceps brachii tendon (star). H indicates humerus.

In conclusion, surgically proven distal pectoralis major tendon avulsions and tears most commonly showed fluid collections at the musculotendinous junction and not at the humeral shaft, with either a normal tendon or hypoechoic tendonlike tissue over the biceps brachii tendon. These findings may potentially cause misdiagnosis of distal tendon avulsions and tears as musculotendinous injuries.

\section{References}

1. Berson BL. Surgical repair of pectoralis major rupture in an athlete: case report of an unusual injury in a wrestler.AmJ Sports Med 1979; 7:348-351.

2. McEntire JE, Hess WE, Coleman SS. Rupture of the pectoralis major muscle: a report of eleven injuries and review of fifty-six.J Bone Joint Surg Am 1972; 54:1040-1046.

3. Wolfe SW, Wickiewicz TL, Cavanaugh JT. Ruptures of the pectoralis major muscle: an anatomic and clinical analysis. Am J Sports Med 1992; 20:587-593

4. Connell DA, Potter HG, Sherman MF, Wickiewicz TL. Injuries of the pectoralis major muscle: evaluation with MR imaging. Radiology 1999; 210:785-791.

5. Lee J, Brookenthal KR, Ramsey ML, Kneeland JB, Herzog R. MR imaging assessment of the pectoralis major myotendinous unit: an MR imaging-anatomic correlative study with surgical correlation. AJRAm JRoentgenol 2000; 174:1371-1375.

6. Weaver JS, Jacobson JA, Jamadar DA, Theisen SE, Ebrahim F, KalumeBrigido M. Sonographic findings of pectoralis major tears with surgical, clinical, and magnetic resonance imaging correlation in 6 patients. JUltrasound Med 2005; 24:25-31.

7. Kretzler HH Jr, Richardson AB. Rupture of the pectoralis major muscle. AmJ Sports Med 1989; 17:453-458.
8. Garrett WE Jr. Muscle strain injuries: clinical and basic aspects. Med Sci Sports Exerc 1990; 22:436-443.

9. Park JY, Espiniella JL. Rupture of pectoralis major muscle: a case report and review of literature. J Bone Joint Surg Am 1970; 52:577-581.

10. Dodds SD, Wolfe SW. Injuries to the pectoralis major. Sports Med 2002; 32:945-952.

11. Quinlan JF, Molloy M, Hurson BJ. Pectoralis major tendon ruptures: when to operate. BrJ Sports Med 2002; 36:226-228.

12. He ZM, Ao YF, Wang JQ, Hu YL, Yin Y. Twelve cases of the pectoralis major muscle tendon rupture with surgical treatment-an average of 6.7year follow-up. Chin Med J (Engl) 2010; 123:57-60.

13. Beloosesky Y, Grinblat J, Katz M, HendelD, Sommer R. Pectoralis major rupture in the elderly: clinical and sonographic findings. Clin Imaging 2003; 27:261-264. 